\title{
西红花酸二甲酯电子吸收光谱与热力学性质的理论研究
}

\author{
聂 汉 李 权* 赵可清 \\ (四川师范大学化学与材料科学学院 “先进功能材料”四川省高校重点实验室 成都 610068)
}

\begin{abstract}
摘要 使用密度泛函理论 B3LYP 方法, 对西红花酸二甲酯的结构、电子吸收光谱、热力学性质进行理论计算研究, 并 基于 Tomasi 的极化统一场模型(PCM)讨论溶剂效应. 结果显示, 溶剂对西红花酸二甲酯的前线分子轨道特征几乎无影 响, 溶剂作用使该分子的最大吸收波长红移约 $42 \mathrm{~nm}$, 红移程度与溶剂极性无关. 西红花酸二甲酯分子的气态热力学性 质与温度的关系式分别为: $C_{\mathrm{mp}}^{\ominus}=189.782+0.925 T, S_{\mathrm{m}}^{\ominus}=457.503+1.366 T, H_{\mathrm{m}}^{\ominus}=1164.899+0.195 T+4.671 \times 10^{-4} T^{2}$, $G_{\mathrm{m}}^{\ominus}=1164.899-0.263 T-8.989 \times 10^{-4} T^{2} .298 \mathrm{~K}$ 时, 西红花酸二甲酯分子的气态标准摩尔生成焓和标准摩尔生成自由 能分别为 -1180.67 和 $-733.23 \mathrm{~kJ} \cdot \mathrm{mol}^{-1}$.
\end{abstract}

关键词 西红花酸二甲酯; 热力学性质; 吸收光谱; 前线分子轨道; 密度泛函理论

\section{Theoretical Studies on Electronic Absorption Spectra and Thermodynamic Properties of Crocetin Dimethyl Ester}

\author{
Nie, Han Li, Quan* Zhao, Keqing \\ (Key Laboratory of Advanced Functional Materials, Sichuan Province Higher Education System, \\ College of Chemistry and Material Science, Sichuan Normal University, Chengdu 610068)
}

\begin{abstract}
The structures, electronic absorption spectra and thermodynamic properties of crocetin dimethyl ester were calculated by using the density functional theory B3LYP method. Then polarized continuum models of tomasi (PCM) have been used to discuss solvent effects. The computational results showed that solvents have no influence on frontier molecular orbital features of crocetin dimethyl ester and solvents action made the maximum absorption wavelength red-shifted about $42 \mathrm{~nm}$. The relationship formulas between temperature and the gaseous thermodynamic properties of crocetin dimethyl ester are as follows: $C_{\mathrm{mp}}^{\ominus}=189.782+0.925 T, \quad S_{\mathrm{m}}^{\ominus}=457.503+1.366 T, \quad H_{\mathrm{m}}^{\ominus}=1164.899+0.195 T+4.671 \times 10^{-4} T^{2}, G_{\mathrm{m}}^{\ominus}=1164.899-$ $0.263 T-8.989 \times 10^{-4} T^{2}$. The standard molar formed enthalpy and free energy of crocetin dimethyl ester molecular are respectively -1180.67 and $-733.23 \mathrm{~kJ} \cdot \mathrm{mol}^{-1}$ at $298.15 \mathrm{~K}$.
\end{abstract}

Keywords crocetin dimethyl ester; thermodynamic property; absorption spectrum; frontier molecular orbital; density functional theory

中草药有效成分的研究在新药研究和开发中起着 非常重要的作用. 西红花又名番红花或藏红花, 是一种 名贵中药材. 西红花酸作为西红花的活性成分之一, 具 有抗氧化、有效降低高胆固醇、保护肝脏、抑制肿瘤等 药理作用, 属于类胡萝卜素物质, 医疗价值极高 ${ }^{[1 \sim 6]}$. 由于西红花室内栽培普遍产生 “萎花” 现象且易感染植 物病毒, 因此以柱头入药的西红花面临着资源短缺的严 峻难题, 难以满足市场的需求. 为了解决这一难题, 科 研人员已通过化学合成方法直接获得西红花酸 ${ }^{[4,7]}$. 西
红花酸二甲酯又叫 $\gamma$-西红花酸, 既是西红花的有效成分 之一，又是合成西红花酸及多种类胡萝卜素的中间体， 越来越受到人们的重视 ${ }^{[1,4,7]}$. 然而对西红花酸二甲酯的 研究鲜见, 尤其未见西红花酸二甲酯的理论研究报道. 因此，本文对西红花酸二甲酯的电子光谱和热力学性质 等进行理论计算, 计算结果有望对深入认识西红花酸二 甲酯及西红花酸及其衍生物的化学合成提供有价值的 信息.

\footnotetext{
*E-mail: liquan6688@163.com

Received July 5, 2011; revised September 1, 2011; accepted September 19, 2011.

Project supported by the National Natural Science Foundation of China (No. 50973076), the Science and Technology Program of Sichuan Province (No. 2010JY0041), and the Scientific Reaserch Foundation of Sichuan Normal University (Nos. 09ZDL03, 025156).

国家自然科学基金(No. 50973076)、四川省科技计划(No. 2010JY0041)和四川师范大学科研基金(Nos. 09ZDL03, 025156)资助项目.
} 


\section{1 计算方法}

采用密度泛函理论在 $\mathrm{B} 3 \mathrm{LYP} / 6-311 \mathrm{G} * *$ 理论水平 上 $^{[8 \sim 10]}$ 对西红花酸二甲酯分子的几种可能结构进行优 化, 得到相应的稳定结构, 比较优化能量获得其最稳定 结构. 在此基础上, 基于频率计算获得不同温度下的热 力学性质及该分子的标准摩尔热力学性质. 根据自洽反 应场(SCRF)理论极化统一场模型(PCM)模型, 采用含时 密度泛函理论(TD-DFT)计算该分子在不同溶剂下的前 线分子轨道和电子吸收光谱，讨论溶剂效应.

\section{2 结果与讨论}

\section{1 结构分析}

根据文献[2，3]报道，西红花酸二甲酯在自然界中 存在如图 1(a)所示的反式结构和如图 1(b)所示的顺式结 构. 在 B3LYP/6-311G**水平上分别对其反式和顺式结 构进行优化, 得到顺式结构的优化能量比反式结构高 $4.81 \mathrm{~kJ} \cdot \mathrm{mol}^{-1}$, 由此可知反式结构比顺式结构更稳定. 其反式结构为 $C_{2 h}$ 对称性, 左右两部分的结构参数相同, 结构参数见表 1 . 由文献[11]得知: 碳碳单键和碳碳双 键的平均键长分别为 0.1541 和 $0.1337 \mathrm{~nm}$. 反式结构中 的碳碳单键明显缩短, 碳碳双键略有增长, 单、双键键 长的差别缩小, 有平均化的倾向, 这符合共轭多烯的基 本结构特征. 在优化得到的顺式结构中, 键角 C(4')$\mathrm{C}\left(5^{\prime}\right)-\mathrm{C}\left(6^{\prime}\right), \mathrm{C}\left(5^{\prime}\right)-\mathrm{C}\left(6^{\prime}\right)-\mathrm{C}\left(7^{\prime}\right), \mathrm{C}\left(6^{\prime}\right)-\mathrm{C}\left(7^{\prime}\right)-\mathrm{C}\left(8^{\prime}\right)$ 和 $\mathrm{C}\left(7^{\prime}\right)-\mathrm{C}\left(8^{\prime}\right)-\mathrm{C}\left(9^{\prime}\right)$ 分别为 $125.9^{\circ}, 122.4^{\circ}, 128.4^{\circ}$ 和 $123.3^{\circ}$ ，与反式结构比较，除 $\mathrm{C}\left(5^{\prime}\right)-\mathrm{C}\left(6^{\prime}\right)-\mathrm{C}\left(7^{\prime}\right)$ 角度外， 其余角度基本一致. 本文还设计出西红花酸二甲酯的另 一种同分异构体, 结构式如图 1(c), 并在相同的理论水 平进行结构优化计算, 得到优化能量比反式结构高 1.40 $\mathrm{kJ} \bullet \mathrm{mol}^{-1}$ ，其稳定性介于反式与顺式结构之间，因此, 西红花酸二甲酯主要以反式结构存在, 本文以此结构为 基础进行相关计算.<smiles>COC(=O)/C(C)=C/C=C/C(C)=C/C=C/C=C(C)/C=C/C=C(\C)C(=O)OC</smiles>

(a)<smiles>COC(=O)C(C)=C/C=C/C(C)=C/C=C/C=C(C)/C=C/C=C(\C)C(=O)OC</smiles>

(b)<smiles>C/C=C/C=C(C)/C(C)=C/C=C/C=C/C=C/C(C)=C/C=C(\C)C(=O)OC</smiles>

图 1 西红花酸二甲酯的异构体结构式

Figure 1 Isomers structural formula of the crocetin dimethyl ester
表 1 反式结构的结构参数

Table 1 Structure parameters of the trans structure

\begin{tabular}{cccc}
\hline Bond & Length/nm & Bond & Angle $/\left(^{\circ}\right)$ \\
\hline $\mathrm{C}(1)-\mathrm{C}(2)$ & 0.1488 & $\mathrm{C}(1)-\mathrm{C}(2)-\mathrm{C}(3)$ & 115.8 \\
$\mathrm{C}(2)-\mathrm{C}(3)$ & 0.1356 & $\mathrm{C}(2)-\mathrm{C}(3)-\mathrm{C}(4)$ & 127.7 \\
$\mathrm{C}(3)-\mathrm{C}(4)$ & 0.1436 & $\mathrm{C}(3)-\mathrm{C}(4)-\mathrm{C}(5)$ & 122.2 \\
$\mathrm{C}(4)-\mathrm{C}(5)$ & 0.1359 & $\mathrm{C}(4)-\mathrm{C}(5)-\mathrm{C}(6)$ & 126.7 \\
$\mathrm{C}(5)-\mathrm{C}(6)$ & 0.1444 & $\mathrm{C}(5)-\mathrm{C}(6)-\mathrm{C}(7)$ & 118.0 \\
$\mathrm{C}(6)-\mathrm{C}(7)$ & 0.1369 & $\mathrm{C}(6)-\mathrm{C}(7)-\mathrm{C}(8)$ & 128.1 \\
$\mathrm{C}(7)-\mathrm{C}(8)$ & 0.1429 & $\mathrm{C}(7)-\mathrm{C}(8)-\mathrm{C}(9)$ & 123.5 \\
$\mathrm{C}(8)-\mathrm{C}(9)$ & 0.1364 & & \\
\hline
\end{tabular}

\section{2 前线分子轨道与电子吸收光谱}

基于自洽反应场(SCRF)理论 PCM 模型，采用 TDB3LYP 方法和 6-311G*, 6-311G**, 6-311+ $\mathrm{G}^{*}, 6-311+$ $+G^{* *}$ 基组分别计算西红花酸二甲酯在甲醇溶剂下的 HOMO, LUMO 轨道本征能量和电子吸收光谱中的最大 吸收波长，结果见表 2 .

表 2 甲醇溶剂中的西红花酸二甲酯在不同基组下的 HOMO, LUMO 能量 $\left(\mathrm{kJ} \cdot \mathrm{mol}^{-1}\right)$ 及能级差 $\left[\Delta_{\mathrm{L}-\mathrm{H}} /\left(\mathrm{kJ} \cdot \mathrm{mol}^{-1}\right)\right]$ 和最大吸收波 长 $\left(\lambda_{\max } / \mathrm{nm}\right)$

Table 2 Energies $\left(\mathrm{kJ} \cdot \mathrm{mol}^{-1}\right)$ of HOMO and LUMO orbitals, energy-level differences $\left[\Delta_{\mathrm{L}-\mathrm{H}} /\left(\mathrm{kJ} \cdot \mathrm{mol}^{-1}\right)\right]$ and maximum absorption wavelengths $\left(\lambda_{\max } / \mathrm{nm}\right)$ on the different standard basis of the crocetin dimethyl ester in methanol solvent

\begin{tabular}{lrrrc}
\hline & $6-311 \mathrm{G}^{*}$ & $6-311 \mathrm{G}^{* *}$ & $6-311+\mathrm{G}^{*}$ & $6-311++\mathrm{G}^{* *}$ \\
\hline$E_{\mathrm{HOMO}}$ & -507.27 & -506.98 & -514.02 & -514.41 \\
$E_{\mathrm{LUMO}}$ & -270.09 & -270.01 & -279.83 & -280.43 \\
$\Delta_{\mathrm{L}-\mathrm{H}}$ & 237.18 & 236.97 & 234.19 & 233.98 \\
$\lambda_{\max }$ & 519.00 & 519.60 & 528.80 & 529.50 \\
\hline
\end{tabular}

由表 2 列出的数据可知，在基组 6-311G*和 6$311 \mathrm{G}^{* *}, 6-311+\mathrm{G}^{*}$ 和 $6-311++\mathrm{G}^{* * 下}$ 的计算结果很接 近，说明计算时对氢原子添加弥散函数和极化函数几乎 不影响西红花酸二甲酯的前线轨道本征能量和电子吸 收光谱. 随着基组的增大，该化合物分子在甲醇溶剂下 的 HOMO 轨道能量、 LUMO 能量、HOMO 与 LUMO 能级差和最大吸收波长的计算结果的改变值均减小，可 以认为在基组 6-311+ $+\mathrm{G}^{* *}$ 下的计算结果已经收敛, 故选取 6-311++G**基组，使用 TD-B3LYP 方法分别 计算西红花酸二甲酯在气相和四氯化碳 $\left(\mathrm{CCl}_{4}\right)$ 、二氯甲 烷 $\left(\mathrm{CH}_{2} \mathrm{Cl}_{2}\right)$ 、丙酮 $\left(\mathrm{CH}_{3} \mathrm{COCH}_{3}\right)$ 、甲醇 $\left(\mathrm{CH}_{3} \mathrm{OH}\right)$ 溶剂下的 50 个单重一单重激发态，以此来讨论溶剂效应.

由图 2 可以看出, 西红花酸二甲酯无论在气相还是 在溶剂相中, HOMO 电子云主要分布在共轭成键 $\pi$ 轨道 上, LUMO 电子云主要集中在共轭反键 $\pi$ *轨道上, HOMO 到 LUMO 的电子跃迁属于 $\pi \rightarrow \pi *$ 跃迁, 溶剂对该 分子 $\mathrm{HOMO}-1, \mathrm{HOMO}, \mathrm{LUMO}, \mathrm{LUOMO}+1, \mathrm{LUMO}+$ 
2 特征几乎无影响. 图 3 为计算得到的西红花酸二甲酯 在气相和 $\mathrm{CCl}_{4}$ 等 4 种溶剂下紫外-可见吸收光谱图, 表 3 分别列出气相和 4 种溶剂下的电子光谱数据 $(\varepsilon$ 为溶剂 相对介电常数, $\lambda$ 为吸收波长, $f$ 为振子强度, $E_{\mathrm{g}}$ 为垂直跃 迁能, 主要的轨道跃迁贡献 Main contribution). 由图 3 和表 3 显示, 西红花酸二甲酯在 4 种溶剂下的紫外-可见 吸收光谱图几乎完全重合, 说明溶剂的极性对该分子电 子吸收光谱的影响可以忽略. 西红花酸二甲酯无论在气 相状态还是在溶剂下的最大吸收波长即为最低能量吸
收波长, 主要来自于 $\mathrm{HOMO}$ 到 LUMO 的电子跃迁，且 在气相和 4 种溶剂下的最大吸收波长分别位于 491 和 $533 \mathrm{~nm}$ 左右, 说明溶剂效应使最大吸收波长红移约 42 $\mathrm{nm}$, 且与溶剂极性无关. 溶剂作用有利于西红花酸二甲 酯分子的 $\mathrm{S}_{0} \rightarrow \mathrm{S}_{1}$ 的电子跃迁.

\section{3 热力学性质}

为了预测西红花酸二甲酯 $\left(\mathrm{C}_{22} \mathrm{H}_{28} \mathrm{O}_{4}\right)$ 分子 $298 \mathrm{~K}$ 时 的气态标准摩尔生成焓 $\Delta_{\mathrm{f}} G_{\mathrm{m}}^{\ominus}$ 和气态标准摩尔生成自由 能 $\Delta_{\mathrm{f}} G_{\mathrm{m}}^{\ominus}$ 等热力学参数, 设计反应如下:

表 3 西红花酸二甲酯在气相和不同溶剂下的电子吸收光谱数据

Table 3 The electronic absorption spectrum data of the crocetin dimethyl ester in gas and different solvents

\begin{tabular}{|c|c|c|c|c|c|c|}
\hline Solvent & $\varepsilon$ & Electronic transition & $\lambda / \mathrm{nm}$ & $f$ & $E_{\mathrm{g}} /\left(\mathrm{kJ} \cdot \mathrm{mol}^{-1}\right)$ & Main contribution \\
\hline \multirow[t]{7}{*}{ Gas } & & $\mathrm{S}_{0} \rightarrow \mathrm{S}_{1}$ & 491 & 3.0438 & 243.14 & $\mathrm{HOMO} \rightarrow \mathrm{LUMO}(71 \%)$ \\
\hline & & $\mathrm{S}_{0} \rightarrow \mathrm{S}_{7}$ & 292 & 0.1480 & 410.06 & $\mathrm{HOMO}-1 \rightarrow \mathrm{LUMO}+1(59 \%)$ \\
\hline & & & & & & $\mathrm{HOMO} \rightarrow \mathrm{LUMO}+2(32 \%)$ \\
\hline & & & & & & HOMO $-2 \rightarrow$ LUMO (7\%) \\
\hline & & $\mathrm{S}_{0} \rightarrow \mathrm{S}_{12}$ & 257 & 0.2225 & 465.06 & $\mathrm{HOMO}-1 \rightarrow \mathrm{LUMO}+1(27 \%)$ \\
\hline & & & & & & $\mathrm{HOMO} \rightarrow \mathrm{LUMO}+2(24 \%)$ \\
\hline & & & & & & $\mathrm{HOMO}-2 \rightarrow \mathrm{LUMO}(17 \%)$ \\
\hline \multirow[t]{7}{*}{$\mathrm{CCl}_{4}$} & 2.23 & $\mathrm{~S}_{0} \rightarrow \mathrm{S}_{1}$ & 534 & 3.1418 & 223.85 & $\mathrm{HOMO} \rightarrow \mathrm{LUMO}(77 \%)$ \\
\hline & & $\mathrm{S}_{0} \rightarrow \mathrm{S}_{7}$ & 294 & 0.1370 & 407.17 & $\mathrm{HOMO}-1 \rightarrow \mathrm{LUMO}+1(57 \%)$ \\
\hline & & & & & & $\mathrm{HOMO} \rightarrow \mathrm{LUMO}+2(33 \%)$ \\
\hline & & & & & & HOMO $-2 \rightarrow$ LUMO (8\%) \\
\hline & & $\mathrm{S}_{0} \rightarrow \mathrm{S}_{10}$ & 268 & 0.2816 & 446.73 & $\mathrm{HOMO}-1 \rightarrow \mathrm{LUMO}+1(30 \%)$ \\
\hline & & & & & & $\mathrm{HOMO} \rightarrow \mathrm{LUMO}+2(23 \%)$ \\
\hline & & & & & & HOMO $-2 \rightarrow$ LUMO (18\%) \\
\hline \multirow[t]{7}{*}{$\mathrm{CH}_{2} \mathrm{Cl}_{2}$} & 8.93 & $\mathrm{~S}_{0} \rightarrow \mathrm{S}_{1}$ & 535 & 3.1374 & 223.85 & $\mathrm{HOMO} \rightarrow \mathrm{LUMO}(76 \%)$ \\
\hline & & $\mathrm{S}_{0} \rightarrow \mathrm{S}_{5}$ & 296 & 0.1408 & 404.27 & $\mathrm{HOMO}-1 \rightarrow \mathrm{LUMO}+1(58 \%)$ \\
\hline & & & & & & $\mathrm{HOMO} \rightarrow \mathrm{LUMO}+2(31 \%)$ \\
\hline & & & & & & HOMO $-2 \rightarrow$ LUMO (9\%) \\
\hline & & $\mathrm{S}_{0} \rightarrow \mathrm{S}_{9}$ & 269 & 0.2769 & 445.76 & $\mathrm{HOMO}-1 \rightarrow \mathrm{LUMO}+1(30 \%)$ \\
\hline & & & & & & $\mathrm{HOMO} \rightarrow \mathrm{LUMO}+2(23 \%)$ \\
\hline & & & & & & $\mathrm{HOMO}-2 \rightarrow \mathrm{LUMO}(20 \%)$ \\
\hline \multirow[t]{7}{*}{$\mathrm{CH}_{3} \mathrm{COCH}_{3}$} & 20.7 & $\mathrm{~S}_{0} \rightarrow \mathrm{S}_{1}$ & 531 & 3.1287 & 224.81 & $\mathrm{HOMO} \rightarrow \mathrm{LUMO}(76 \%)$ \\
\hline & & $\mathrm{S}_{0} \rightarrow \mathrm{S}_{5}$ & 296 & 0.1430 & 404.27 & $\mathrm{HOMO}-1 \rightarrow \mathrm{LUMO}+1(58 \%)$ \\
\hline & & & & & & $\mathrm{HOMO} \rightarrow \mathrm{LUMO}+2(30 \%)$ \\
\hline & & & & & & HOMO $-2 \rightarrow$ LUMO (9\%) \\
\hline & & $\mathrm{S}_{0} \rightarrow \mathrm{S}_{9}$ & 268 & 0.2704 & 446.73 & $\mathrm{HOMO}-1 \rightarrow \mathrm{LUMO}+1(29 \%)$ \\
\hline & & & & & & $\mathrm{HOMO} \rightarrow \mathrm{LUMO}+2(22 \%)$ \\
\hline & & & & & & HOMO $-2 \rightarrow$ LUMO $(20 \%)$ \\
\hline \multirow[t]{7}{*}{$\mathrm{CH}_{3} \mathrm{OH}$} & 32.63 & $\mathrm{~S}_{0} \rightarrow \mathrm{S}_{1}$ & 530 & 3.1240 & 225.78 & $\mathrm{HOMO} \rightarrow \mathrm{LUMO}(76 \%)$ \\
\hline & & $\mathrm{S}_{0} \rightarrow \mathrm{S}_{5}$ & 296 & 0.1441 & 404.27 & $\mathrm{HOMO}-1 \rightarrow \mathrm{LUMO}+1(59 \%)$ \\
\hline & & & & & & $\mathrm{HOMO} \rightarrow \mathrm{LUMO}+2(30 \%)$ \\
\hline & & & & & & HOMO $-2 \rightarrow$ LUMO (9\%) \\
\hline & & $\mathrm{S}_{0} \rightarrow \mathrm{S}_{9}$ & 267 & 0.2673 & 447.69 & $\mathrm{HOMO}-1 \rightarrow \mathrm{LUMO}+1(29 \%)$ \\
\hline & & & & & & $\mathrm{HOMO} \rightarrow \mathrm{LUMO}+2(22 \%)$ \\
\hline & & & & & & HOMO $-2 \rightarrow$ LUMO $(20 \%)$ \\
\hline
\end{tabular}




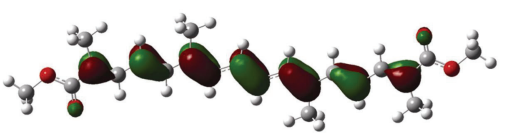

$\mathrm{HOMO}$ in gas

go

LUMO in carbontetrachloride

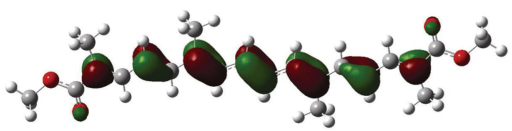

HOMO in acetone $\left(\mathrm{CH}_{3} \mathrm{COCH}_{3}\right)$

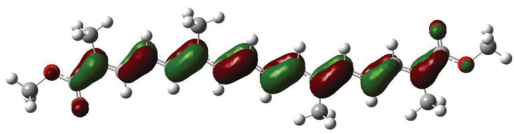

LUMO in methanol

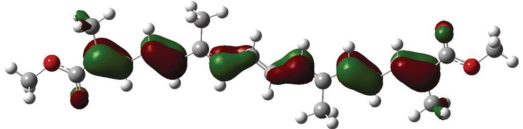

$\mathrm{HOMO}-1$ in carbontetrachloride

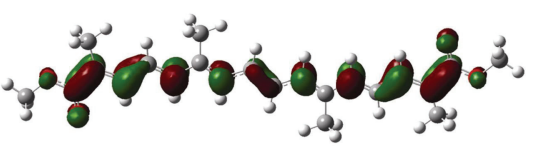

LUMO +1 in dichloromethane

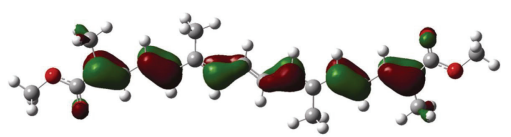

HOMO -1 in methanol

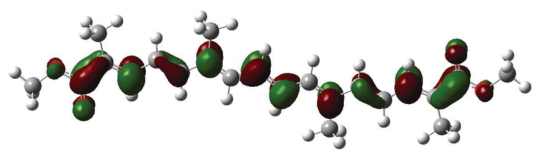

$\mathrm{LUMO}+2$ in gas

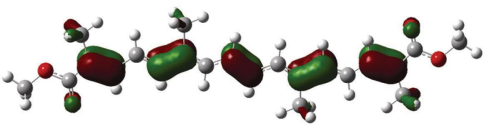

HUMO -2 in dichloromethane

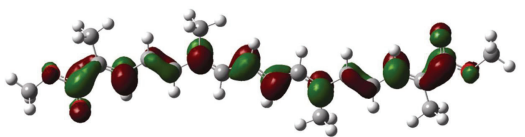

$\mathrm{LUMO}+2$ in acetone
ว

LUMO in gas

कृ

$\mathrm{HOMO}$ in dichloromethane $\left(\mathrm{CH}_{2} \mathrm{Cl}_{2}\right)$

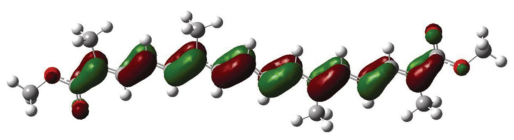

LUMO in acetone

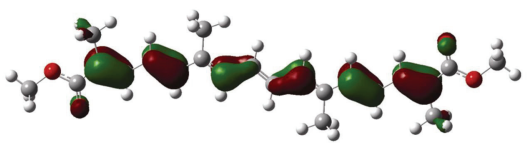

HOMO -1 in gas

-

LUMO +1 in carbontetrachloride

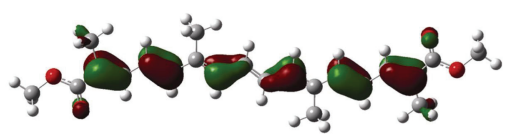

HOMO -1 in acetone

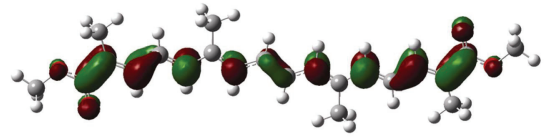

$\mathrm{LUMO}+1$ in methanol

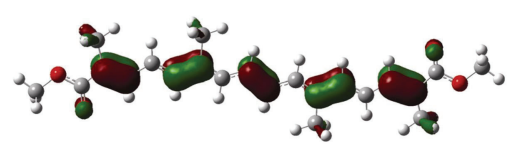

HUMO-2 in carbontetrachloride

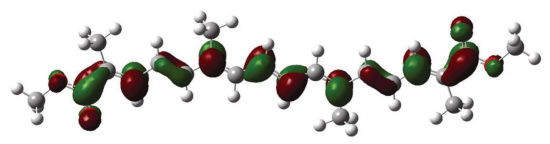

LUMO +2 in dichloromethane

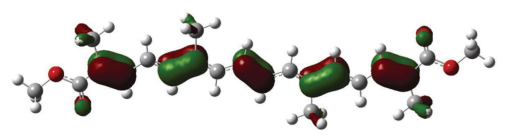

HUMO -2 in methanol

\section{og}

$\mathrm{HOMO}$ in carbontetrachloride $\left(\mathrm{CCl}_{4}\right)$

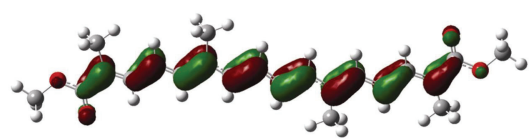

LUMO in dichloromethane

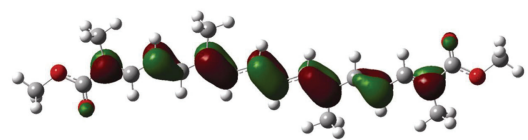

$\mathrm{HOMO}$ in methanol $\left(\mathrm{CH}_{3} \mathrm{OH}\right)$

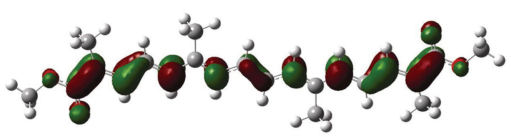

LUMO +1 in gas

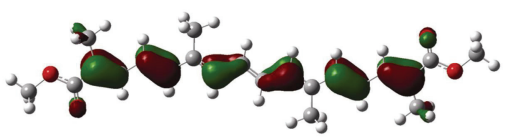

HOMO - 1 in dichloromethane

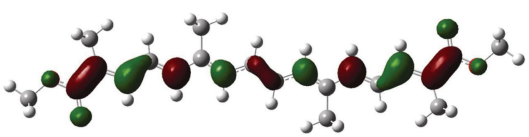

LUMO +1 in acetone

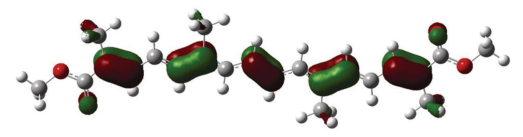

HUMO -2 in gas

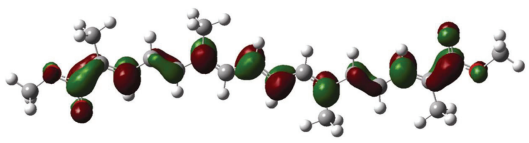

$\mathrm{LUMO}+2$ in carbontetrachloride

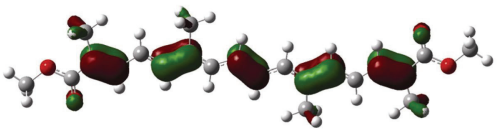

HUMO -2 in acetone

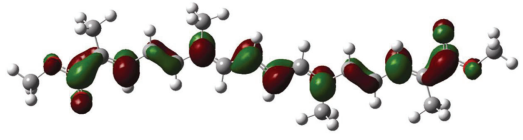

$\mathrm{LUMO}+2$ in methanol

图 2 西红花酸二甲酯的前线分子轨道

Figure 2 Frontier molecular orbitals of the crocetin dimethyl ester

$\mathrm{C}_{22} \mathrm{H}_{28} \mathrm{O}_{4}(\mathrm{~g})+27 \mathrm{O}_{2}(\mathrm{~g}) \rightarrow 22 \mathrm{CO}_{2}(\mathrm{~g})+14 \mathrm{H}_{2} \mathrm{O}(\mathrm{g})$

该反应的标准摩尔反应焓变 $\Delta_{\mathrm{r}} H_{\mathrm{m}}^{\ominus}$ 、标准摩尔摘变

$\Delta_{\mathrm{r}} S_{\mathrm{m}}^{\ominus}$ 、标准摩尔自由能变 $\Delta_{\mathrm{r}} G_{\mathrm{m}}^{\ominus}$ 的计算公式 ${ }^{[12,13]}$ 如下:

$\Delta_{\mathrm{r}} H_{\mathrm{m}}^{\ominus}=\sum\left[v_{\mathrm{A}}(H+E)\right]_{\text {products }}-\sum\left[v_{B}(H+E)\right]_{\text {reactants }}$

$$
\begin{aligned}
& \Delta_{\mathrm{r}} S_{\mathrm{m}}^{\ominus}=\sum\left(v_{\mathrm{A}} S_{\mathrm{m}}^{\ominus}\right)_{\text {products }}-\sum\left(v_{\mathrm{B}} S_{\mathrm{m}}^{\ominus}\right)_{\text {reactants }} \\
& \Delta_{\mathrm{r}} G_{\mathrm{m}}^{\ominus}=\Delta_{r} H_{\mathrm{m}}^{\ominus}-T \Delta_{\mathrm{r}} S_{\mathrm{m}}^{\ominus}
\end{aligned}
$$

式中 $E$ 为气相分子的电子总能量, $H$ 为焓的校正值, $S_{\mathrm{m}}^{\ominus}$ 标准摩尔熵, products 和 reactants 分别表示所有生成物 


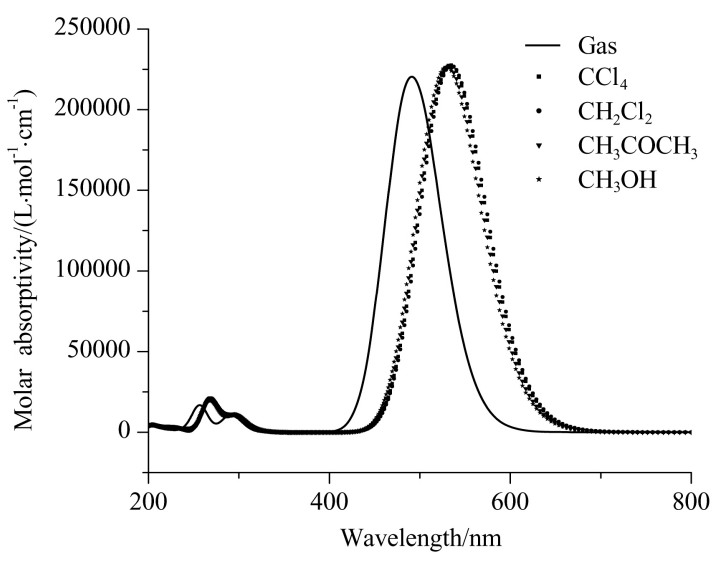

图 3 西红花酸二甲酯在气相和不同溶剂下的紫外-可见吸 收光谱

Figure 3 UV-Vis absorption spectrums of the crocetin dimethyl ester in gas and different solvents

和反应物, $v_{\mathrm{A}}$ 和 $v_{\mathrm{B}}$ 分别表示生成物和反应物的化学计量 数. 在 B3LYP/6-311G**水平上计算得到 $298 \mathrm{~K}$ 、标准压 力下生成物和反应物的热力学参数 $(H+E)$ 和 $S_{\mathrm{m}}^{\ominus}$, 由公 式(2) (4)分别计算得到反应式(1)在 $298 \mathrm{~K}$ 时的标准摩 尔热力学性质 $\Delta_{\mathrm{r}} H_{\mathrm{m}}^{\ominus}, \Delta_{\mathrm{r}} S_{\mathrm{m}}^{\ominus}, \Delta_{\mathrm{r}} G_{\mathrm{m}}^{\ominus}$ 见表 4. 由文献[13] 可查到 $\mathrm{CO}_{2}(\mathrm{~g})$ 的 $\Delta_{\mathrm{f}} H_{\mathrm{m}}^{\ominus}, \Delta_{\mathrm{f}} G_{\mathrm{m}}^{\ominus}$ 分别为 -393.509 , $394.359 \mathrm{~kJ} \bullet \mathrm{mol}^{-1}, \mathrm{H}_{2} \mathrm{O}(\mathrm{g})$ 的 $\Delta_{\mathrm{f}} H_{\mathrm{m}}^{\ominus}, \Delta_{\mathrm{f}} G_{\mathrm{m}}^{\ominus}$ 分别为一 $241.818,-228.572 \mathrm{~kJ} \bullet \mathrm{mol}^{-1}$. 利用公式(5), (6), 可分别 求得西红花酸二甲酯的气态标准摩尔生成焓 $\Delta_{\mathrm{f}} H_{\mathrm{m}}^{\ominus}$ 和气 态标准摩尔生成自由能 $\Delta_{\mathrm{f}} G_{\mathrm{m}}^{\ominus}$, 见表 4. $\Delta_{\mathrm{r}} H_{\mathrm{m}}^{\ominus}, \Delta_{\mathrm{f}} H_{\mathrm{m}}^{\ominus}$, $\Delta_{\mathrm{r}} G_{\mathrm{m}}^{\ominus}$ 和 $\Delta_{\mathrm{f}} G_{\mathrm{m}}^{\ominus}$ 单位分别为 $\mathrm{kJ} \cdot \mathrm{mol}^{-1}, \Delta_{\mathrm{r}} S_{\mathrm{m}}^{\ominus}$ 单位为 $\mathrm{J} \bullet$ $\mathrm{mol}^{-1} \cdot \mathrm{K}^{-1}$.

$$
\begin{aligned}
& \Delta_{\mathrm{r}} H_{\mathrm{m}}^{\ominus}=\sum\left(v_{\mathrm{A}} \Delta_{\mathrm{f}} H_{\mathrm{m}}^{\ominus}\right)_{\text {products }}-\sum\left(v_{\mathrm{B}} \Delta_{\mathrm{f}} H_{\mathrm{m}}^{\ominus}\right)_{\text {reactants }} \\
& \Delta_{\mathrm{r}} G_{\mathrm{m}}^{\ominus}=\sum\left(v_{\mathrm{A}} \Delta_{\mathrm{f}} G_{\mathrm{m}}^{\ominus}\right)_{\text {products }}-\sum\left(v_{\mathrm{B}} \Delta_{\mathrm{f}} G_{\mathrm{m}}^{\ominus}\right)_{\text {reactants }}
\end{aligned}
$$

表 4 西红花酸二甲酯的标准热力学性质

Table 4 Standard thermodynamic properties of the crocetin dimethyl ester

\begin{tabular}{cccccc}
\hline$\Delta_{\mathrm{r}} H_{\mathrm{m}}^{\ominus}$ & $\Delta_{\mathrm{r}} S_{\mathrm{m}}^{\ominus}$ & $\Delta_{\mathrm{r}} G_{\mathrm{m}}^{\ominus}$ & $\Delta_{\mathrm{f}} H_{\mathrm{m}}^{\ominus}$ & $\Delta_{\mathrm{f}} G_{\mathrm{m}}^{\ominus}$ & $S_{\mathrm{m}}^{\ominus}$ \\
\hline-10861.98 & 941.45 & -11142.67 & -1180.67 & -733.24 & 866.63 \\
\hline
\end{tabular}

由表 4 得知, 在 $298 \mathrm{~K}$ 、标准大气压下, 西红花酸二 甲酯的生成反应是放热的自发反应.

为了定量描述西红花酸二甲酯的热力学性质与温 度的关系, 计算该分子在不同温度和标准压力下的标准 摩尔焓 $H_{\mathrm{m}}^{\ominus}$ 、标准摩尔等压热容 $C_{\mathrm{mp}}^{\ominus}$ 、标准摩尔熵 $S_{\mathrm{m}}^{\ominus}$, 并进行拟合, 得到相应的关系, 如图 4 所示.

定量描述式如下:

$$
C_{\mathrm{mp}}^{\ominus}=189.782+0.925 T\left(\mathrm{~J} \cdot \mathrm{mol}^{-1} \cdot \mathrm{K}^{-1}\right)
$$
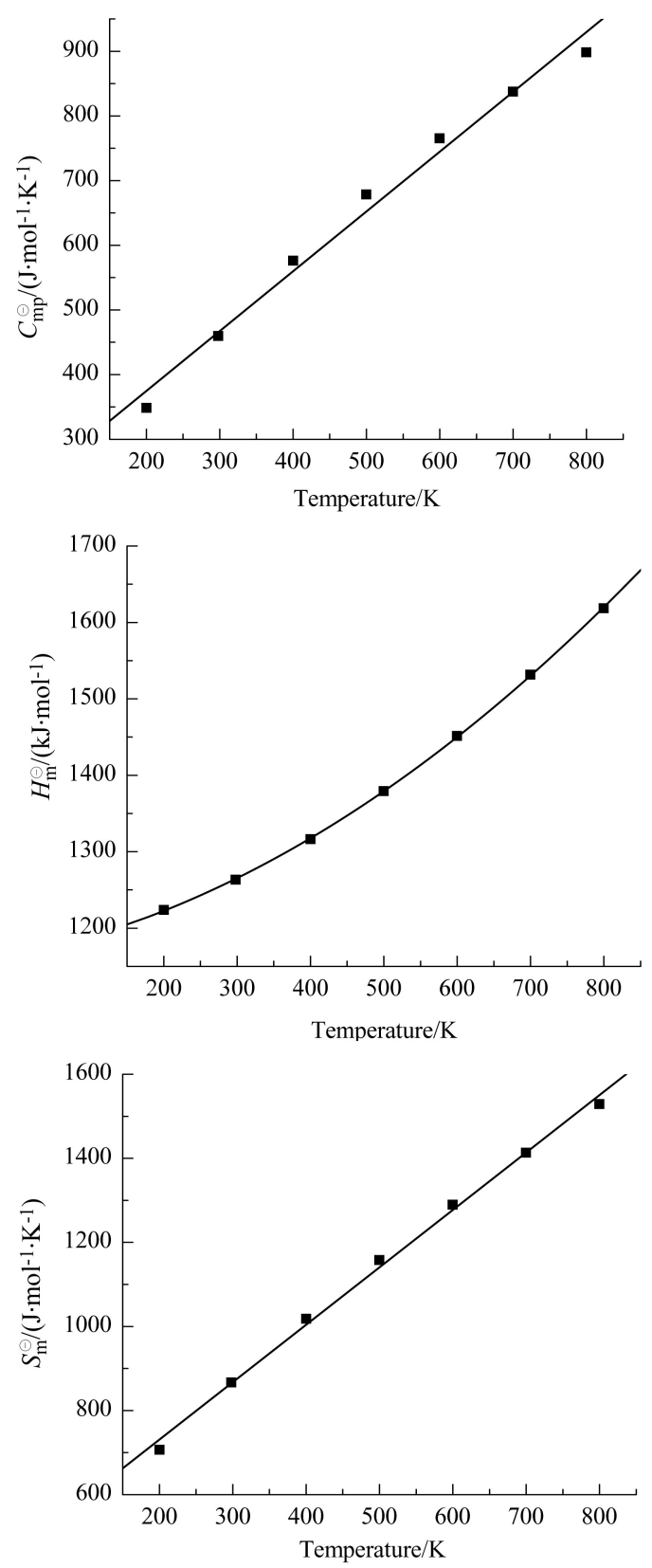

图 4 西红花酸二甲酯分子的热力学函数与温度的关系

Figure 4 Relationship between temperature and thermodynamic functions of the crocetin dimethyl ester

$$
\begin{aligned}
& H_{\mathrm{m}}^{\ominus}=1164.899+0.195 T+4.671 \times 10^{-4} T^{2}\left(\mathrm{~kJ} \cdot \mathrm{mol}^{-1}\right) \\
& S_{\mathrm{m}}^{\ominus}=457.503+1.366 T\left(\mathrm{~J} \cdot \mathrm{mol}^{-1} \cdot \mathrm{K}^{-1}\right)
\end{aligned}
$$

由公式 $G_{\mathrm{m}}^{\ominus}=H_{\mathrm{m}}^{\ominus}-T S_{\mathrm{m}}^{\ominus}$ 可得到 $G_{\mathrm{m}}^{\ominus}$ 与温度的关系式 为:

$G_{\mathrm{m}}^{\ominus}=1164.899-0.263 T-8.989 \times 10^{-4} T^{2}\left(\mathrm{~kJ} \cdot \mathrm{mol}^{-1}\right)$

如图 4 所示, 随着温度升高, 西红花酸二甲酯的气 态热力学性质 $H_{\mathrm{m}}^{\ominus}$ 呈二次曲线增大, $C_{\mathrm{mp}}^{\ominus}$ 和 $S_{\mathrm{m}}^{\ominus}$ 呈线性 增大，这是由于温度越高分子振动加剧. 此外, 其气态 热力学性质 $G_{\mathrm{m}}^{\ominus}$ 随着温度的升高而降低. 


\section{3 结论}

本文采用密度泛函理论 B3LYP 方法对西红花酸二 甲酯分子的结构、前线分子轨道、电子吸收光谱和热力 学性质进行理论计算研究, 得到如下结论: (1)溶剂对西 红花酸二甲酯的前线分子轨道特征几乎无影响. 溶剂效 应使该分子的最大吸收波长红移约 $42 \mathrm{~nm}$, 红移程度与 溶剂的极性无关. (2)在 $298 \mathrm{~K}$ 时, 西红花酸二甲酯分子 的气态标准摩尔热力学性质 $\Delta_{\mathrm{f}} H_{\mathrm{m}}^{\ominus}, \Delta_{\mathrm{f}} G_{\mathrm{m}}^{\ominus}$ 和 $S_{\mathrm{m}}^{\ominus}$ 分别为 $-1180.67 \mathrm{~kJ} \cdot \mathrm{mol}^{-1},-733.23 \mathrm{~kJ} \cdot \mathrm{mol}^{-1}$ 和 $866.63 \mathrm{~J} \bullet$ $\mathrm{mol}^{-1} \cdot \mathrm{K}^{-1}$. 随着温度升高, 西红花酸二甲酯的气态热力 学性质 $H_{\mathrm{m}}^{\ominus}$ 呈二次增大, $C_{\mathrm{mp}}^{\ominus}$ 和 $S_{\mathrm{m}}^{\ominus}$ 呈线性增大, $G_{\mathrm{m}}^{\ominus}$ 呈 二次曲线降低.

\section{References}

[1] Fang, Z. K.; Wang, J. X. Nat. Prod. Res. Dev. 2007, 19, 280 (in Chinese).

(方志凯, 王建新, 天然产物研究与开发, 2007, 19, 280.)

[2] Li, N.; Lin, G.; Chiou, G. C. Y.; Min, Z. D. J. Chin. Pharm. Univ. 1999, 30, 108 (in Chinese).

(李娜, 林鸽, Geroge C. Y. Chiou, 闵知大, 中国药科大学学报, 1999, 30, 108.)

[3] Zhang, H.; Zhang, X. S.; Yan, F.; Zeng, Y. H.; Chen, F.; Wei, M. Chem. Res. Appl. 2000, 12, 487 (in Chinese).

(张宏, 张新申, 颜钫, 曾宇红, 陈放, 魏明, 化学研究与应用, 2000, 12, 487.)

[4] Zhang, H.; Zhang, J. G.; Zhang, L.; Yan, F.; Chen, F. Chem. Res. Appl. 2009, 21, 794 (in Chinese).
(张宏, 张俊国, 张伶, 颜钫, 陈放, 化学研究与应用, 2009, 21, 794.)

[5] Li, W. N.; Qian, Z. Y. Chin. J. New. Drugs 2005, 14, 1165 (in Chinese). (李文娜, 钱之玉, 中国新药杂志, 2005, 14, 1165.)

[6] Zhang, J. G. M.S. Thesis, Sichuan Normal University, Chengdu, 2009, p. 27 (in Chinese). (张俊国, 硕士论文, 四川师范大学, 成都, 2009, p. 27.)

[7] Zhang, J. G.; Zhang, H.; Zhang, L.; Yan, F.; Chen, F. Acta Chim. Sinica 2008, 66, 1451 (in Chinese). (张俊国, 张宏, 张伶, 颜钫, 陈放, 化学学报, 2008, 66, 1451.)

[8] Yang, F.; Xu, B. Y.; Li, Q.; Zhao, K. Q.; Fan, Z. J. J. Atom Mol. Phys. 2010, 27, 605 (in Chinese)

(杨帆，徐布一，李权，赵可清，范志金，原子与分子物理学报， 2010, 27, 605.)

[9] Liu, F. Y.; Hu, J. D.; Li, Q.; Zhao, K. Q. Chin. J. Org. Chem. 2007, 27, 507 (in Chinese). (刘焕英，胡兢丹，李权，赵可清，有机化学, 2007, 27, 507.)

[10] Li, X. M.; Zhang, J. P. Acta Phys. Sin. 2010, 59, 7736 (in Chinese). (李雪梅, 张建平, 物理学报, 2010, 59, 7736.)

[11] Wang, X. L. Organic Chemistry, Higher Education Press, Beijing, $\mathbf{2 0 0 5}$, p. 4 (in Chinese). (汪小兰，有机化学，高等教育出版社，北京, 2005, p. 4.)

[12] Huang, H. S.; Zhang, T. L.; Zhang, J. G.; Wang, L. Q.; Yang, L.; Qiao, X. J.; Shang, J. Acta Chim. Sinica 2010, 68, 289 (in Chinese). (黄辉胜, 张同来, 张建国, 王丽琼, 杨利, 乔小晶, 尚静, 化学 学报, 2010, 68, 289.)

[13] Fu, X. C.; Shen, W. X.; Yao, T. Y.; Hou, W. H. Physical Chemistry, Higher Education Press, Beijing, 2005 (in Chinese). (傅献彩，沈文霞，姚天扬，侯文华，物理化学，高等教育出版 社, 北京, 2005.) 